\title{
SEXUAL DIMORPHISM OF PELVIC MORPHOLOGY VARIATION IN LIVE HUMANS
}

\author{
Oksana Kolesova, Janis Vètra \\ Riga Stradins University, Latvia
}

\begin{abstract}
This study represents the comparison of the morphology variation in different planes of female and male pelvis. Anthropological literature represents two different views of the pelvic morphology variation. On the one hand, the variation is considered lower in females than in males. On the other hand, some empirical findings demonstrated no differences in the pelvic morphology variation between sexes. Moreover, some measures of female pelvis demonstrate higher coefficients of variation. Taking into account that previous findings were based on linear measures, it seems important to analyze pelvic proportions and the variation of the inlet, middle, and outlet planes.

The study was based on the retrospective pelvimetry of threedimensional computer tomography of 176 live males and 212 females. Anterioposterior diameters and transverse diameters were measured in four planes, and their ratios were calculated in order to evaluate proportions and variances. The Levene's test for the equality of variances was used to evaluate the observed variation in male and female pelvis morphology.

The results confirmed well established sexual dimorphism in the pelvic linear measures - the anteroposterior diameters of the midplane and the outlet. In addition, this study identified higher variation in the transverse diameter of the inlet in females. The proportions demonstrated no differences in variation in all the planes but the midplane including bispinous diameter $(\mathrm{F}=11.34 ; \mathrm{p}<.01)$. It seems important that the female pelvis cavity demonstrated lower variance than the male pelvis cavity in the midplane including bispinous. This finding supports the view of selective pressure on the female pelvis and its intensity in the midplane.
\end{abstract}

Key words: pelvic morphology, sexual dimorphism, pelvimetry. 


\section{INTRODUCTION}

Evolutionarily, the human pelvis has adapted to two processes which changed the morphology of its cavity. Primarily, the human pelvis was shaped by the erect posture and the bipedal locomotion [1]. It became more triangular because of the increased transverse diameter of the inlet, widening and the enlargement of the sacrum, and the shortening of the pubic symphisis. Secondarily, the female pelvis was exposed to an additional selective pressure of the obstetric difficulties caused by the encephalization of a newborn $[1,7,10]$. As a result, the shape of the female pelvic cavity became shorter and more cylindrical. The observed pelvic sexual dimorphism is genetically determined and modulated by the steroid influence on the pelvic growth [10]. An additional impact on the pelvic shape is related to the early physical work and early pregnancy [1].

Anthropological literature represents two different views of the pelvic morphology variation. Some authors considered that an additional evolutionary pressure on the female pelvis results in the lower variation in its morphology in comparison to the male pelvis [6]. Tague [9], in its turn, has found no differences in the variation between males and females. Moreover, some pelvic dimensions demonstrated higher variation in females. It should be noted that these conclusions were based on the variances of the linear measures made on skeletal collections. The aim of this study was to analyze the differences of live humans' pelvic morphology variation in males and females with focusing on the proportions of lesser pelvic planes.

It is possible to analyze at last the four planes: the inlet, two midplanes, and the outlet. The inlet plane includes the linea terminalis. The first midplane is formed by the midpoint of pubic simphysis, two acetabulum centers, and a joint between the second and the third sacral vertebras. It is the wider plane of the pelvic cavity. The second midplane is formed by the lower border of pubic simphysis, two ishial spines, and a joint between the fourth and the fifth sacral vertebras. It is the shorter plane of the pelvic cavity. The outlet plane is formed by the lower border of pubic simphysis, pubic rami, ischial tuberosities and the tip of coccyx.

It should be noted that the indexes of proportions - the ratios of linear pelvic measures - are not widely applied in the research on sexual dimorphism. Taking into account that the human pelvis' shape can be 
represented as a closed ring with a cylindrical cavity, the analysis of pelvic proportions can be helpful in the description of pelvic planes and in the comparisons of pelvic shapes without analyzing other general anthropometric measures (e.g. height). Research on the variation of these proportions can adds to understanding of pelvic morphology in females and males. Three-dimensional pelvimetry in live humans, in its turn, can provide good visualization of bones and a high accuracy of measures [2].

The research question was: What are the differences in the variation of proportions of the inlet, middle, and outlet pelvic planes and the corresponding linear pelvic measures?

\section{MATERIAL AND METHODS}

The study was based on the archive data of the Department of Radiology, "Gailezers" Hospital, Latvia. The measures were based on the pelvic images performed in the period from October 2009 to November 2010. Archive data were available according to legal requirements. The research sample included 122 females aged from 18 to 84 (the mean age $=48.1, \mathrm{SD}=18.3$ years) and 176 males aged from 18 to 82 (the mean age $=43.6, \mathrm{SD}=16.1$ ). Exclusion criteria were bones' fractures, osteoporosis, scoliosis, and polytraumas.

Three-dimensional multiplanar reconstruction was performed on $1.25 \mathrm{~mm}$ slices. The anteroposterior diameter and the transverse diameter of each plane were detected.

(1) Diameters of the inlet: the anteroposterior diameter - the distance between the posterosuperior border of the pubic simphysis and the promontory of the sacrum; the transverse diameter - the maximum distance between iliopectineal lines;

(2) Diameters of the midplane including biacetabular: the anteroposterior diameter - the distance between the posterior midpoint of the pubic simphysis and the border of the second and the third sacral vertebrae; the transverse diameter (biacetabular) - the distance between the middle of acetabulums;

(3) Diameter of the midplane including bispinous: the anteroposterior diameter - the distance between the lower border of pubic simphysis and anterior fourth and fifth sacral vertebrae; the transverse diameter (bispinous) - the lowest distance between two ischial spines; 
(4) Diameters of the outlet: the anteroposterior diameter - the distance between the lower border of pubic simphysis and the tip of coccyx; the transverse diameter (bituberous) - the maximum distance between the two internal points of ischial tuberosities.

Indexes of proportions in each plane were calculated as the ratio of the anteroposterior and the transverse diameter. The data analysis was performed using PASW 18.0 program in order to compute descriptive statistics, the Levene's test for the equality of variance, and the $t$-test.

\section{RESULTS}

Table 1 demonstrates the descriptive statistics of linear measures, the indexes of pelvic planes, and the inferential statistics concerning the comparison of means and the variances of these measures. All the linear measures of the lesser pelvis represent sexual dimorphism with higher means in females than in males. The most visible differences are observed on bispinous $(\mathrm{t}=20.85, \mathrm{p}<.001)$ and bituberous $(\mathrm{t}=20.72$, $\mathrm{p}<.001)$. According to the Levene's test for equality of variances, the variances of linear pelvic measures statistically differ in three dimensions: the transverse diameter of inlet $(\mathrm{F}=5.54, \mathrm{p}<.01)$, the anteroposterior diameter of midplane $(\mathrm{F}=4.82, \mathrm{p}<.01)$, and the anteroposterior diameter of outlet $(\mathrm{F}=17.33, \mathrm{p}<.001)$. For all of them the variance is higher in females than in males. 
Table 1. Summary of descriptive and inferential statistics for pelvic dimensions and their variance

\begin{tabular}{|c|c|c|c|c|c|c|}
\hline \multirow[b]{2}{*}{ Measures } & \multicolumn{2}{|c|}{ Males $(n=176)$} & \multicolumn{2}{|c|}{$\begin{array}{l}\text { Females } \\
(n=212)\end{array}$} & \multirow{2}{*}{ 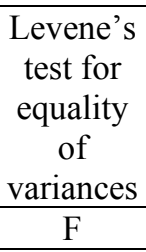 } & \multirow{2}{*}{$\frac{\mathrm{t} \text {-test }}{\mathrm{t}}$} \\
\hline & $X$ & SD & $\mathrm{X}$ & SD & & \\
\hline \multicolumn{7}{|l|}{ Inlet diameters } \\
\hline Anteroposterior & 119.23 & 10.16 & 124.18 & 10.26 & 0.08 & $5.39^{* * *}$ \\
\hline Transverse & 126.82 & 7.49 & 135.08 & 8.49 & $5.34^{*}$ & $10.44^{* * *}$ \\
\hline \multicolumn{7}{|l|}{$\begin{array}{l}\text { Midplane } 1 \\
\text { diameters }\end{array}$} \\
\hline Anteroposterior & 127.78 & 8.98 & 131.46 & 9.84 & 1.26 & $3.87^{* * *}$ \\
\hline Biacetabular & 113.82 & 7.21 & 122.23 & 8.33 & 2.61 & $10.63^{* * *}$ \\
\hline \multicolumn{7}{|l|}{$\begin{array}{l}\text { Midplane } 2 \\
\text { diameters }\end{array}$} \\
\hline Anteroposterior & 116.48 & 7.49 & 122.52 & 8.74 & $4.82^{*}$ & $7.22^{* * * *}$ \\
\hline Bispinous & 93.64 & 8.64 & 112.31 & 9.27 & 2.01 & $20.85^{* * *}$ \\
\hline \multicolumn{7}{|l|}{ Outlet diameters } \\
\hline Anteroposterior & 95.97 & 7.38 & 99.69 & 9.78 & $17.33^{* * *}$ & $4.22^{* * *}$ \\
\hline Bituberous & 103.48 & 9.33 & 124.18 & 10.39 & 2.30 & $20.72^{* * *}$ \\
\hline \multicolumn{7}{|l|}{$\begin{array}{l}\text { Indexes of } \\
\text { proportion }\end{array}$} \\
\hline Inlet & 0.94 & 0.08 & 0.93 & 0.10 & 3.37 & -1.54 \\
\hline Midplane1 & 1.13 & 0.10 & 1.08 & 0.10 & 0.07 & $-4.67^{* * *}$ \\
\hline Midplane2 & 1.25 & 0.14 & 1.10 & 0.10 & $11.34^{* *}$ & $-12.72^{* * * *}$ \\
\hline Outlet & 0.94 & 0.11 & 0.81 & 0.11 & 0.02 & $-11.24^{* * *}$ \\
\hline
\end{tabular}

${ }^{* * *}-\mathrm{p}<.001{ }^{* *}-\mathrm{p}<.01$.

Indexes of proportions demonstrate that there are no significant differences in the proportions of the inlet plane. The comparison demonstrates that the transverse diameter is higher than the anteroposterior diameter for both sexes. There are also no differences in variance in this plane.

In the midplane, including biacetabular, proportion indexes statistically differ for both sexes $(\mathrm{t}=-4.67, \mathrm{p}<.001)$ without differences in their variance. The index is greater than 1.00 in females and males. It 
means that the anteroposterior diameter is greater than the transverse diameter. The female pelvic cavity is wider and rounder because the index is closer to 1.00 than in males.

In the midplane including bispinous, indexes are statistically higher for males and females $(\mathrm{t}=12.72, \mathrm{p}<.001)$. This plane demonstrates also statistically significant differences in variance $(\mathrm{F}=11.34, \mathrm{p}<.01)$. The index of this plane is greater than 1.00 for both sexes. The anteroposterior diameter is greater than the transverse diameter. This plane has also a rounder shape with a wider cavity and a lower variation in the proportion in females.

The outlet indexes are statistically different $(\mathrm{t}=-11.24, \mathrm{p}<.001)$ without differences in variance. The outlet is wider in females. At the same time, the index is less than 1.00, and the transverse diameter is wider than the anteroposterior diameter for both sexes.

\section{DISCUSSION}

In general, the results of this study demonstrate significant differences in the variation of linear pelvic measures and their proportions in females and males. The higher variation was detected on the anteroposterior diameter in the midplane of the pelvic cavity including bispinous. At the same time, the proportion in this plane is less variable in females than in males. Higher variation was detected also on the variation in the transverse diameter of the inlet plane and in the anteroposterior diameter of the outlet plane without significant differences in the variation of proportions of these planes.

These findings need to be discussed by taking into account the findings on variation in the anteroposterior diameter of the midplane and the outlet of the female pelvis presented by Tague [9]. In accordance with Tague, higher variability in lower pelvic planes is determined by the effect of relaxin secreted during pregnancy that increases the mobility of pelvic joints. During delivery sacral nutation, the anteroposterior diameter of the outlet can increase by 10 to $20 \mathrm{~mm}$ [3].

In line with Tague's findings, the present study demonstrates that the anteroposterior diameters of the midplane and the outlet are more variable in females than in males. In addition, higher variability in the transverse diameter of the inlet was found. This is a new finding that was not observed in previous studies $[6,9,10]$. The observed 
differences in the variance of the transverse diameter can be explained by a hormonal effect at the end of pregnancy, which leads to softening of the pubic simphysis. This process allows the pubic bones to move apart $1 \mathrm{~cm}$ that increases pelvic diameters [5]. It is possible to consider the effect of relaxin as being the principal determinant to higher variability in lower pelvic planes as also in the inlet.

One more explanation is related to differences in materials under investigation. This study was based on pelvimetry in live humans, but Tague's and Meindl's studies [9, 6] were based on the measurements of skeletal collections without a compensation of pelvic joints (e.g. simphyseal disk).

Another explanation of higher variations in linear measures in females addresses to the human birth mechanism. During delivery the sagital suture of the fetal head is placed in the transverse diameter of the inlet and the anteroposterior diameter of the outlet. It is possible to suppose that this process has an impact on the higher variation in these dimensions. This point requests an additional research because the findings demonstrated higher sexual dimorphism in the anteroposterior diameter of the inlet, the transverse diameter of the midplane, and the transverse diameter of the outlet, which are related to a biparietal deformation during delivery [4].

According to Schultz [7], the pressure of selection on the female pelvis is related to the process of fetal encephalization, which impacted the inlet and the midplane of pelvis. The present study also confirms differences in proportions of pelvic planes. It seems important that the most visible sexual dimorphism is observed in the midplane, whereas the inlet proportions do not differ in males and females. This finding is in accordance with Tague [10], who classified the anteroposterior and the transverse diameters of the inlet as being nondimorfic.

In the present study, the midplane, including biacetabular, demonstrates no differences in the sense of variation. In comparison to bispinous, the biacetabular diameter demonstrates a lower variability in both sexes [9]. This tendency is interpreted as the adaptation to the erectal posture when the weight is distributed along the alae of sacrum and through the ischial tuberosites towards the acetabulum [5]. Therefore, the main adaptation in the human pelvis in this dimension is related to the erect posture and bipedality. 
Concerning the midplane including bispinous, the most important finding represents the lower variation of its proportion in females than in males. The observed variation of this index is lower despite the higher variation of the anteroposterior diameter in females than in males. It is possible to suppose that the linear measures of this plane have higher correlation in females (i.e. the higher anteroposterior diameter of midplane is related to the higher bispinous diameter). The mean index of the proportion is 1.08 . Therefore, the shape of female pelvis in this plane is near to round. In males, the anteroposterior diameter is less related to the bispinous diameter that results in the higher variation in the shape of the pelvic cavity. According to the evolutionary biology, there is an inverse relationship between the intensity of stabilizing selection and the variance in phenotypes (and genotypes) within a population [8]. Lower variation in the morphology of the midplane - the narrowest plane of the female pelvis - confirms higher selective pressure addressing to the process of human birth.

To sum up, this study demonstrates significant sexual differences in the variability of pelvic dimensions. The suggested focusing on the indexes of proportions was useful in the identification of the differences in the midplane. These results support the view on the intensity of the selection intensity as inversely related to phenotypic variability.

\section{REFERENCES}

1. Abitbol M.M. (1987) Obstetrics and posture in pelvic anatomy. Journal of Human Evolution, 16, 243-255.

2. Anderson N., Humphries N., Wells J. (2005) Measurement error in computed tomography pelvimetry. Australasian Radiology, 49, 104107.

3. Borell U., Fernström I. (1957) The movements at the sacro-iliac joints and their importance to changes in the pelvic dimentions during parturition. Acta Obstet. Gynecol. Scand. 36, 42-57.

4. Correia H., Balseiro S., De Areia M. (2005) Sexual dimorphism in the human pelvis: Testing a new hypothsis. Journal of Comparative Human biology, 56, 153-160.

5. Kapandji I.A. (2008) The physiology of the joint. Vol. 3. The spinal column, pelvic girdle and head. Edinburg: Elselvier Limited. 
6. Meindl R.S., Lovejoy C.O., Mensforth R.P., Don Carlos L. (1985) Accuracy and direction of error in the sexing of the skeleton: Implication for paleodemography. Amer J Phys Anthropol, 68, 79-85.

7. Schultz A.H. (1949) Sex differences in the pelvis of primates. Amer J Phys Anthropol, 7, 401-423.

8. Stansfield W.D. (1977) The Science of Evolution. New York: Macmillan.

9. Tague R.G. (1989) Variation in pelvic size between males and females. Amer J Phys Anthropol, 80, 59-71.

10. Tague R.G. (1992) Sexual dimorphism in the human bony pelvis, with a consideration of the Neandertal pelvis from Kebara Cave, Israel. Amer J Phys Anthropol, 88, 1-21.

\section{Address for correspondence:}

Oksana Kolesova,

Riga Stradins University

Lacplesa iela, 43/45-10, Riga, LV-1011, Latvia

E-mail: oksana-kolesova@inbox.lv 